\title{
Más allá del clientelismo y la técnica: la distribución política del presupuesto gubernamental en Colombia
}

DOI: https://doi.org/10.18046/recs.i35.4745

\section{Beyond Clientelism and Technique: The Political Distribution of Government Budget in Colombia}

\author{
Santiago Leyva-Botero ${ }^{* *}$ \\ Universidad EAFIT (Medellín, Colombia) \\ Claudia Mejía-Betancur ${ }^{* * *}$ \\ Universidad EAFIT (Medellín, Colombia) \\ Jose Antonio Fortou ${ }^{* * *}$ \\ Universidad EAFIT (Medellín, Colombia)
}

\begin{abstract}
* Este artículo de investigación científica y tecnológica es producto final del proyecto de investigación "La distribución del presupuesto discrecional en Colombia: un análisis desde la política distributiva", financiado por Colciencias y la Universidad EAFIT (Colombia) bajo la modalidad de Jóvenes Investigadores e Innovadores en 2014-2015. El proyecto fue desarrollado en el Centro de Análisis Político y el Grupo de Investigación Sociedad, Política e Historias Conectadas, adscritos al Departamento de Gobierno y Ciencias Políticas de la Universidad EAFIT. Un borrador de este artículo fue presentado en el VIII Congreso Latinoamericano de Ciencia Política, organizado por la Asociación Latinoamericana de Ciencia Política en julio de 2015 en Lima, Perú. Agradecemos al estudiante de Economía de la Universidad EAFIT Esteban Alemán por su colaboración en la recopilación de datos; a la estudiante de Ciencias Políticas de la Universidad EAFIT María Bran por su asistencia en la organización del texto final; y a los profesores Orlando Espinosa Santiago (Benemérita Universidad Autónoma de Puebla, México) y Luis de la Calle (Centro de Investigación y Docencia Económicas de la Ciudad de México, México) por sus valiosos comentarios a versiones preliminares de este artículo. La base de datos y el código utilizado para analizarla estarán disponibles en un repositorio público de la plataforma GitHub (www.github. com/josefortou). Artículo de investigación recibido el 06.04.2021 y aceptado el 24.09.2021.

${ }^{* *}$ Doctor en Management (Public Governance) de la Lancaster University (Inglaterra). Profesor titular del Departamento de Gobierno y Ciencias Políticas y coordinador de la Maestría en Gobierno y Políticas Públicas de la Universidad EAFIT (Colombia). Correo electrónico: sleyvabo@eafit.edu.co ORCID: https://orcid.org/oooo-ooo1-5940-1413

*** Magíster en Gobierno y Políticas Públicas de la Universidad EAFIT (Colombia). Joven Investigadora Colciencias (2015), miembro del grupo de investigación Sociedad, Política e Historias Conectadas de la Universidad EAFIT. Correo electrónico: cmejiab@gmail.com ORCID: https://orcid.org/oooo-ooo2-0481-1623
\end{abstract}


**** Doctor en Ciencia Política de The Ohio State University (Estados Unidos). Profesor Auxiliar del Departamento de Gobierno y Ciencias Políticas y jefe del pregrado en Ciencias Políticas, Universidad EAFIT (Colombia). Autor responsable y de correspondencia. Correo electrónico: jfortour@eafit.edu.co ORCID: https://orcid.org/oooo-0oo2-3127-4288

\section{Cómo citar/How to cite}

Leyva-Botero, Santiago; Mejía-Betancur, Claudia; Fortou, Jose Antonio (2021). Más allá del clientelismo y la técnica: la distribución política del presupuesto gubernamental en Colombia.

Revista CS, 35, 125-153. https://doi.org/10.18046/recs.i35.4745 


\section{Resumen}

En este artículo examinamos la distribución presupuestal por el ejecutivo central en un régimen presidencialista desde la literatura sobre la distribución táctica. Nuestro objetivo es evaluar empíricamente teorías existentes sobre la racionalidad política de este fenómeno. Iniciamos con una revisión de la literatura comparada, con énfasis en la distribución orientada hacia votantes y miembros del legislativo. Derivamos tres hipótesis que capturan tres modelos teóricos distintos: distribución técnica, distribución hacia votantes y distribución hacia el legislativo. Posteriormente, analizamos una base de datos original sobre la distribución de cupos indicativos presupuestales en Colombia durante el primer gobierno del presidente Juan Manuel Santos (2010-2014). Mediante un análisis de regresión, mostramos que los incrementos en el grado de representación en el congreso se asocian positivamente con el monto de las asignaciones presupuestales. Este hallazgo da soporte empírico a teorías sobre la política distributiva no programática orientada al legislativo.

\section{PALABRAS CLAVE:}

presupuesto del Estado, congreso, presidencialismo, política distributiva, Colombia

We examine the distribution of budget by the national executive power in a presidential system from the standpoint of the literature on tactical distribution. Our objective is to empirically test theories on the political rationale of this phenomenon. We begin with a comparative literature review, emphasizing voter and congress-oriented distribution. We derive three hypotheses that capture three distinct models: programmatic, voter-oriented, and legislative-oriented distribution. Then, we analyze an original dataset of budget distribution through cupos indicativos in Colombia during president Juan Manuel Santos' first term (2010-2014). Using regression analyses, we show that increases in legislative representation have a positive association with the amount of resources distributed, which lend empirical support to theories of nonprogrammatic legislative-oriented distributive politics.

\section{KEYWORDS:}

State Budget, Congress, Presidentialism, Distributive Politics, Colombia 



\section{Introducción}

En 2011, tras la aprobación de la Ley de Regalías en Colombia, el entonces ministro de hacienda Juan Carlos Echeverri señaló que con este nuevo sistema de transferencias presupuestales sería posible "repartir la mermelada en toda la tostada nacional" (“Aprobada la reforma a las regalías", 2011). Esta frase llevó a que, desde entonces, el uso del término mermelada se convirtiera en un sinónimo de la distribución de recursos del presupuesto nacional por parte del presidente con fines políticos. La oposición le dio una connotación negativa al término, asociándolo con intereses electorales, corrupción e intercambios por apoyos legislativos. Así mismo, este vocablo se ha utilizado para denotar la negociación con políticos regionales mediante la asignación de cupos de gasto - conocidos como cupos indicativos en el país-por parte del presidente.

Las profundas diferencias en la asignación de cupos indicativos por departamento en Colombia invitan a examinar la distribución del presupuesto nacional en detalle. Mientras que en su primer periodo el presidente Juan Manuel Santos (2010-2014) no distribuyó recursos por cupos indicativos a departamentos con necesidades objetivas como Chocó, o recursos naturales como Arauca, otros -Antioquia, Atlántico y Córdoba- recibieron sumas elevadas. ¿Qué revelan estas diferencias sobre el uso táctico que da el presidente a recursos discrecionales para poder negociar con élites políticas locales y regionales? ¿Es posible explicar esta distribución del presupuesto de los cupos indicativos desde un punto de vista estratégico?

El objetivo de este artículo es entender este asunto desde la literatura comparada en ciencia política sobre la distribución política de los presupuestos nacionales, globalmente y en Latinoamérica. El fenómeno de la distribución política del presupuesto no es nuevo, ni exclusivo del caso colombiano: términos como pork barrel o électoralisme son utilizados en otros contextos para referirse a este tipo de mecanismos de negociación política a través del presupuesto. De hecho, dentro de la ciencia política existe una literatura alrededor de la incidencia de la política en la distribución del recurso, o política distributiva (Nazareno; Stokes; Brusco, 2006; Stokes; Dunning; Nazareno; Brusco, 2013; Golden; Min, 2013; Hicken; Nathan, 2020). Esta literatura busca examinar y entender los factores que explican la distribución del presupuesto, en especial cuando se puede demostrar que los criterios no son técnicos y que, por el contrario, se recurre a estrategias para mantener el poder, relacionadas con la entrega de algunos incentivos a actores o regiones que puedan beneficiar los intereses de quien asigna los recursos.

Esta literatura se ha desarrollado principalmente en países como Estados Unidos, cuyo sistema electoral mayoritario y uninominal, unido al bipartidismo y la burocra- 
cia institucionalizada, devienen en el uso de estrategias diferentes a las que pueden utilizarse en un contexto como el latinoamericano o colombiano. Sin embargo, los trabajos sobre este tema se han globalizado con estudios de China (Jiang; Zhang, 2020) y el Medio Oriente (Blaydes, 2020), entre otros. Así mismo, existen referentes importantes en las democracias presidencialistas de Latinoamérica. En los últimos 30 años han aparecido trabajos sobre Argentina, México y Brasil que han incorporado variables como la representación política en el legislativo (Porto; Sanguinetti, 1996; González, 2012; Calvo; Murillo, 2004; González; Mamone, 2012; González; Mamone, 2015), la política social (Garay; Palmer-Rubin; Poertner, 2020) y la filiación partidista (Díaz-Cayeros; Estévez; Magaloni, 2016), los cuales han permitido avanzar en la comprensión del fenómeno de la política distributiva.

Sin embargo, estos trabajos analizan países con un régimen federal que otorga un amplio poder a los niveles intermedios de gobierno. Esto genera la oportunidad de explorar si estas teorías, en conversación con aquellas desarrolladas en Estados Unidos, pueden ayudar a explicar el proceso de distribución política de recursos en un país unitario y presidencial como Colombia. De tal forma, este artículo se une a una pequeña literatura sobre el uso de mecanismos tácticos de distribución presidencial en Colombia, distinta al cuerpo de estudios robusto sobre clientelismo en el país, que sigue vigente hoy (González-Tule; Guzmán-Mendoza; Tuirán-Sarmiento, 2021). Se destacan trabajos como el de Mejía-Guinand, Botero y Rodríguez-Raga (2008) sobre los Consejos Comunales de Gobierno durante el gobierno de Álvaro Uribe (2002-2010), en el cual analizan el gasto en infraestructura vial desde la literatura sobre política distributiva. Por su parte, Leyva-Botero (2012) hace un análisis en clave de relaciones intergubernamentales de los mismos Consejos Comunales. Recientemente, Benson (2021) ha enlazado los ciclos electorales con la redistribución estratégica de tierra en el país. En comparación, los cupos indicativos del período de Santos (2010-2018) han sido poco estudiados. Valencia y Ávila (2014) analizan los mismos datos que utilizamos en este artículo, pero de manera descriptiva y con el fin de evidenciar estrategias electorales por parte de congresistas, no del presidente. En años más recientes, Bonilla-Mejía e Higuera-Mendieta (2017) y Rodríguez-Valero y Maldonado-Gómez (2020) han estudiado este fenómeno, pero desde perspectivas económica y normativa, respectivamente. En consecuencia, todavía tenemos mucho por explorar en cuanto al alcance de la distribución presupuestal en Colombia como mecanismo táctico. De ahí el valor de tomar los únicos datos conocidos sobre la distribución política del presupuesto durante el periodo presidencial de Santos y explorarlos con más detalle bajo el enfoque que proponemos.

En este artículo realizamos un análisis estadístico de la distribución departamental de los cupos indicativos entre los congresistas de la coalición del gobierno 
Santos. Para ello construimos una base de datos original que utiliza información filtrada a la prensa sobre cupos indicativos por departamento, así como estadísticas oficiales. Mostramos que la distribución de recursos por cupos indicativos tiene una asociación positiva y significativa con la representación legislativa. Este hallazgo sugiere que el presidente utiliza una estrategia de distribución presupuestal basada en privilegiar a los votantes fieles (los core voters), mediante la negociación con élites políticas regionales. En esto, coincidimos con Mejía-Guinand et al. (2008) cuando concluyen que los criterios políticos pesan más que los técnicos en el momento de explicar la distribución del gasto a nivel territorial en Colombia. De esta manera, aportamos elementos importantes para entender el funcionamiento del régimen político y territorial colombiano. Así mismo, los hallazgos sobre el caso colombiano nos ayudan a pensar y repensar la política distributiva en otros sistemas presidenciales y unitarios en la región.

Iniciamos el artículo con una revisión de la literatura sobre política distributiva y presupuesto táctico para contextualizar la discusión y la manera en la que abordamos el problema. Después, plantearemos una serie de hipótesis derivadas de los principales modelos teóricos en la literatura. Posteriormente, discutiremos la estrategia empírica que utilizamos para evaluar estas hipótesis, incluyendo la selección de casos, variables y fuentes de información; y para terminar, presentaremos y discutiremos los resultados estadísticos para aportar a la comprensión del fenómeno desde una perspectiva explicativa a la luz del contexto colombiano.

\section{¿Por qué los presidentes recurren a la distribución táctica del presupuesto?}

Explicar la distribución del presupuesto por parte del ejecutivo es un asunto central para el estudio de la política. Las estrategias de distribución política del presupuesto por parte del ejecutivo pueden ser utilizadas para garantizar apoyo electoral o legislativo, o incluso para implementar políticas que sean de la preferencia de los electores. En este apartado revisaremos la literatura sobre política distributiva no programática, enfocándonos en teorías sobre la distribución táctica del presupuesto. Sobre este último punto, identificamos dos escuelas. Unos sugieren que la distribución de recursos por parte del ejecutivo se realiza con el fin de movilizar apoyos electorales, sea de votantes leales (core voters) o indecisos (swing voters), con lo que observaríamos una mayor destinación de recursos donde hay un número mayor de estos votantes; mientras otros trabajos argumentan que los presidentes distribuyen recursos a las unidades territoriales más representadas en el legislativo, con el fin 
de mantener lealtades políticas de representantes electos. Contrastaremos estas teorías de la distribución táctica del presupuesto con instancias de distribución programática. Al final, derivaremos hipótesis de cada una de estas tres teorías con el fin de evaluarlas empíricamente.

\section{Política distributiva no programática y presupuesto táctico}

La primera distinción en la literatura sobre política distributiva se presenta entre dos tipos de distribuciones: programática y no programática (Stokes et al., 2013; Hicken, 2011). La distribución programática (o de política pública) es aquella que ocurre bajo un criterio conocido y discutido públicamente para distribuir los recursos del presupuesto. En otras palabras, en este caso la distribución del presupuesto sigue programas de gobierno y se guía por criterios objetivos o necesidad.

La distribución no programática ocurre cuando no existen criterios claros (o transparentes), ni discusión pública al respecto. Esta opacidad puede implicar que sean las razones políticas las que orienten la asignación del gasto público. Este tipo de decisiones de gasto de carácter no programático (y específicamente político), también se conocen en la literatura como presupuesto táctico, término utilizado por Dixit y Londregan (1996) para hablar de recursos que se destinan de manera estratégica para ganar elecciones, como proyectos de construcción y otras estrategias conocidas en Estados Unidos como pork barrel politics. En economía, esta noción ha sido desarrollada desde la literatura sobre ciclos políticos presupuestales y reelección (Rogoff, 1991). Dentro del universo de políticas distributivas no programáticas encontramos dos subtipos (Stokes et al., 2013). En las relaciones clientelares, la recepción del beneficio (el presupuesto) depende del voto de un individuo; pero si no depende del comportamiento electoral, hablamos de sesgo partisano, un tipo que incluye el uso electoral de programas y presupuesto públicos, así como el pork barrel.

En un primer nivel, el grado de politización de la distribución fiscal en un país puede variar de acuerdo con sus instituciones políticas. Por ejemplo, los conflictos previos por el presupuesto y las reglas sobre su uso definen "si la negociación entre políticos [...] es más decisiva que el criterio fiscal" (Gordin, 2009: 7). El régimen político también importa. En los regímenes presidencialistas (en especial aquellos con reelección) el uso del presupuesto táctico por parte del ejecutivo es especialmente importante, dado que una de sus metas principales es la reelección. Este segundo punto implica que los presidentes son actores que están en competencia electoral y se preocupan por la manera como el gasto presupuestal puede beneficiar sus intereses políticos. 
La literatura sugiere que los presidentes pueden usar activamente el presupuesto de manera táctica para resolver algunos de sus problemas en la política electoral, y que lo hacen de manera local o territorial. Los incentivos para dar ese uso político al presupuesto son bastante altos (Mebane; Wawro, 2002): por un lado, los presidentes enfrentan competidores en varios niveles de gobierno que quieren reclamar para sí el crédito por la ejecución de programas y presupuesto; y por otro, la cadena del gasto público, que va desde el presidente en la cúspide hasta el ciudadano, es larga y tenue, lo cual puede generar una falta de claridad sobre a qué actor político se le puede acreditar el gasto. Pero para que el gasto local tenga incidencia en las elecciones presidenciales, las decisiones de inversión que benefician a estos votantes tienen que poder rastrearse hasta el presidente. En estos escenarios, la decisión estratégica para el presidente es o bien hacerle ver a los votantes que el presupuesto les ha llegado por su decisión, o delegarles esta relación a los políticos locales y las élites parlamentarias. En otras palabras, el presidente puede definir mecanismos de asignación directa, o depender de las maquinarias políticas y relaciones clientelares de las élites políticas locales.

A partir de esta idea, Mebane y Wawro (2002) proponen que el gasto presidencial puede ser orientado a los votantes o las élites. En el primer caso, es decir, si los presidentes deciden ser identificados directamente con el gasto, es importante que este sea poco complejo e institucionalizado, para que los votantes puedan ver más fácilmente la intervención directa del presidente (Simison, 2014). Cuando un presidente no puede construir una relación directa con los electores a partir del presupuesto táctico, el gasto se puede orientar a mantener en una coalición a las élites políticas. En la relación entre las élites parlamentarias y el presidente, puede darse el caso en que este les delegue a aquellas la intermediación del presupuesto en las regiones, bien como premio por su apoyo, o como estrategia de convencimiento para que realicen su cambio de bando. De hecho, Simison (2014) muestra que la distribución de recursos en regímenes presidenciales tiende a ser usada para la construcción de coaliciones legislativas: ya que hay menores incentivos que en un régimen parlamentario para crear coaliciones estables, la distribución de recursos se convierte en una estrategia clave. A continuación, redirigimos la distinción entre distribución hacia los votantes y hacia las élites para hablar de dos escuelas teóricas: los que sugieren que los presidentes redirigen el voto pensando directamente en votantes con fines electorales y aquellos que argumentan que lo hacen pensando en el poder legislativo que tienen distintas unidades territoriales y las élites políticas que las representan. 


\section{Votantes leales e indecisos}

Existen diversas explicaciones al problema de la distribución política del presupuesto (Golden; Min, 2013), pero el principal punto de referencia es el debate clásico entre las teorías que señalan que el presidente direcciona el presupuesto táctico hacia los votantes fieles o core voters o hacia los indecisos, los swing voters. En otras palabras, una asignación presupuestal orientada a los votantes. Más recientemente, la llamada hipótesis del campo de batalla electoral se sumó al debate, mostrando otros posibles equilibrios en los que el presidente dirige gasto hacia distritos en los que ambos partidos bordean el $50 \%$ de las preferencias de voto (Larcinese; Snyder; Testa, 2013) o hacia los bastiones de su partido y de la oposición por igual (Horiuchi; Lee, 2008). En otras más, hay una dimensión temporal: Mebane y Wawro (2002) sugieren que, en la primera parte de su período, los presidentes privilegian las élites para compensar su apoyo en las elecciones; mientras que en la segunda mitad privilegian a los votantes, en busca de la reelección. Por último, algunos argumentan que la estrategia es retrospectiva y que la distribución de recursos es una recompensa para los distritos que han sido electoralmente leales (Catalinac; Bueno de Mesquita; Smith, 2020).

En este debate, Cox y McCubbins (1986: 378) proponen que "los políticos (...) invierten poco (si algo) en los grupos de oposición, algo más en los grupos de indecisos, y aún más en los grupos de apoyo". Según esto, a los core voters se les apunta con beneficios privados para retener su lealtad, mientras que otros grupos de votantes serán favorecidos con bienes públicos cuando la lealtad de la base leal del partido es insuficiente para ganar las elecciones (Díaz-Cayeros et al., 2012). Esta tesis de los core voters, que ya había sido debatida en Lindbeck y Weibull (1987) y sigue vigente (Cox, 2010; Stokes, 2005; Stokes et al., 2013), fue confrontada diez años después por Dixit y Londregan (1996), quienes afirman que en contextos en los que han avanzado la burocratización y la consolidación del servicio civil, la asignación del presupuesto empieza a depender más de criterios técnicos y menos particularistas, por lo que resulta mucho más rentable para un gobernante destinar el presupuesto táctico a los swing voters. En otras palabras, la hipótesis de los swing voters asume que el desarrollo de una burocracia moderna y consolidada ha llevado a que las maquinarias políticas dejen de ser útiles como mecanismo distributivo para el presidente.

En Latinoamérica, el debate en torno a las explicaciones de la distribución táctica del presupuesto inicialmente giró en torno a estas dos hipótesis. Así, por ejemplo, en el contexto de los países en desarrollo, la hipótesis de los core voters parece ser más relevante porque la lealtad de los votantes está condicionada a que las maquinarias políticas sigan concediendo sus favores, pues de no hacerlo, los electores son susceptibles a ser movilizados por competidores políticos (Díaz-Cayeros et al., 2012). $\mathrm{Si}$ bien en contextos institucionalizados las inversiones en los core voters pueden 
ser irracionales porque no consideran la condicionalidad de la lealtad partidista, son relevantes en contextos en los que la desinstitucionalización de los partidos imposibilita la construcción de lealtades fuertes (Díaz-Cayeros et al., 2012; Mebane; Wawro, 2002). A su vez esto implica que, en el contexto latinoamericano, el principal reto para los políticos es conservar la lealtad de los core voters, especialmente cuando la posibilidad de ser reelecto está en juego. El trabajo de González y Mamone (2015) muestra que en Argentina y Brasil el gasto presidencial está motivado por consideraciones políticas del tipo core vs. swing. Dado esto, cualquier estrategia por llegar al swing voter podría implicar perder los core voters, cuya lealtad no está asegurada y está condicionada a la continuidad de los incentivos materiales.

\section{Representación política}

En contraposición a estas teorías sobre la distribución no programática con fines electorales y orientada hacia los votantes, han surgido argumentos adicionales a partir del estudio de la representación o sobrerrepresentación de algunos distritos electorales, especialmente en Latinoamérica (Porto; Sanguinetti, 2001; Gibson; Calvo; Falleti, 2004; Calvo; Murillo, 2005), pero también en Estados Unidos (Lee, 200o). La sobre y subrepresentación es una situación común en sistemas electorales proporcionales. Dada la naturaleza inexacta de las fórmulas de traducción de votos en curules, algunas unidades territoriales (provincias, regiones, estados, departamentos, etc.) están sobrerrepresentadas en el legislativo. Esto significa que tienen más poder político que el que deberían tener si solo se tuvieran en cuenta criterios objetivos como el tamaño de su economía, la población o el nivel de pobreza en la misma. Por esta razón, hay incentivos para que el presidente dirija gasto hacia estas unidades y sus élites políticas. Esto conecta con trabajos que muestran cómo los presidentes utilizan la distribución tanto de gasto (pork) como de bienes de coalición (ministerios) para conseguir apoyo legislativo (Raile; Pereira; Power, 2011).

En regímenes federales como los de Argentina y Brasil, parte de la literatura sugiere que la representación política de entidades territoriales explica de manera significativa cómo los presidentes destinan los recursos de su presupuesto táctico: distribuyen más recursos en los lugares más sobrerrepresentados. Pero la eficiencia del gasto táctico en términos electorales aumenta cuando los distritos electorales más pequeños y periféricos son aquellos más sobrerrepresentados, dado que es posible comprar la lealtad de los core voters con relativamente pocos recursos. En efecto, la delegación del gasto a las élites locales cobra mayor importancia cuando un país tiene una cota más alta de periferialización (Gibson; 2012), es decir, cuando "la distribución de las transferencias está sesgada hacia las unidades subnacionales más dependientes de las transferencias" (Gordin, 2009:7). Esta situación se combina 
con la sobrerrepresentación y produce casos en los que la excesiva periferalización, sumada a una alta politización del gasto, termina por generar una descentralización cooptativa en la que el centro maneja cifras relativamente pequeñas del presupuesto, para controlar regiones con alta incidencia en el congreso (Gordin; 2009). La periferialización puede ser mayor en un régimen presidencialista con el nivel de representación de las regiones periféricas en el congreso, dado que esta aumenta los incentivos políticos para que el presidente dirija el presupuesto táctico hacia allí.

En resumen, esta revisión muestra que la utilización del presupuesto y las transferencias discrecionales por parte de los presidentes con fines políticos tácticos es comparativamente común. Si bien existen variadas explicaciones, paro países en desarrollo como los de Latinoamérica hay unas causas más probables. Así mismo, esta revisión ilustra que existen múltiples estrategias y tácticas distributivas que resultan racionales en distintos escenarios, según sea la posibilidad del presidente para reelegirse o para que su sucesor gane en los siguientes comicios. Estas decisiones incluyen las diferentes opciones de priorizar a los votantes leales o apostar por los indecisos. Adicionalmente, la literatura muestra que el bajo desarrollo económico y político condiciona las estrategias distributivas; y de igual modo vemos que distintas instituciones políticas, como el sistema electoral y de partidos y el régimen (presidencial vs parlamentario, federal vs. unitario), también condicionan la elección de bienes públicos o privados para la estrategia distributiva.

\section{Algunas hipótesis}

A partir de la literatura y los diferentes modelos teóricos expuestos, planteamos tres hipótesis sobre la incidencia de distintos factores en la distribución del presupuesto por parte del ejecutivo central a nivel territorial (distritos electorales $u$ otras unidades administrativas subnacionales). Empezamos por una hipótesis derivada de las teorías que suponen una distribución programática del presupuesto:

$\left.H_{r}\right)$ Distribución programática: unidades territoriales con niveles de pobreza más altos reciben una mayor cantidad de recursos.

Si rechazamos esta hipótesis, significa que la distribución del presupuesto tiene una destinación política y no puramente programática o basada en necesidades. En este orden de ideas, derivamos dos hipótesis a partir de teorías sobre la distribución no programática o táctica del presupuesto público por parte del ejecutivo:

$H_{2}$ ) Core voters: unidades territoriales con más partidarios del presidente reciben más recursos. 
Si hay evidencia en esta dirección, podríamos afirmar que existe una distribución táctica (no programática) orientada hacia los core voters como recompensa por su comportamiento electoral pasado, o bien como incentivo frente a una futura elección. Finalmente:

$\left.H_{3}\right)$ Representación política: unidades territoriales con mayor representación en el legislativo (como porcentaje de las curules) reciben más recursos.

Si aceptamos esta última hipótesis, los recursos que distribuye (tácticamente) el presidente serían un elemento de negociación y las unidades territoriales más representadas en el legislativo cobran importancia para extender o mantener el poder presidencial. A continuación, evaluamos de forma empírica estas tres hipótesis.

\section{Métodos: estudiar la distribución táctica del presupuesto en Colombia}

¿Cómo distribuyen el presupuesto los presidentes en sistemas unitarios? En esta sección, buscamos dar respuesta a los interrogantes planteados anteriormente y evaluar las tres hipótesis que derivamos por medio del análisis una base de datos original sobre la distribución geográfica del presupuesto distribuido a los congresistas de la coalición del primer gobierno de Juan Manuel Santos (2010-2014). Para esto, recogimos datos a nivel departamental sobre los montos asignados a estos legisladores por concepto de cupos indicativos, así como información electoral, socioeconómica y demográfica a nivel departamental. Con esta información, estimamos una serie de modelos de regresión lineal que nos permiten evaluar las hipótesis y estimar el efecto de la representación política sobre la distribución táctica del presupuesto.

\section{Variable dependiente: montos por cupos indicativos}

En Colombia, los cupos de gasto asignados por el presidente a legisladores se conocen como cupos indicativos. La importancia de estos cupos se hizo clara desde el inicio del primer período del presidente Santos, cuando se evidenció que había priorizado la figura para darle más juego a los congresistas. El mecanismo como tal había sido creado en el año 2000 por el propio Santos como Ministro de Hacienda del presidente Andrés Pastrana (1998-2002), con el argumento de llevar el presupuesto nacional a las regiones para fomentar su desarrollo. Desde su creación, la figura fue fuente de múltiples debates políticos. Sin embargo, los cupos indicativos, 
a pesar de representar una porción pequeña del presupuesto, son una figura clave para entender las dinámicas de negociación del presidente con el Congreso que le apoyó en la aprobación de reformas tan contenciosas, incluidas las necesarias para aprobar el Acuerdo de Paz con las Fuerzas Armadas Revolucionarias de Colombia (FARC) en 2016.

Medir los recursos destinados a cada departamento por el presidente colombiano es una tarea difícil. La escasez de información en torno a la distribución de cupos se debe a que el Congreso colombiano no tiene iniciativa de gasto ni veto; toda la negociación presupuestal se da manera informal entre el presidente y el Congreso, en especial cuando el primero necesita apoyo legislativo. Formalmente, en términos presupuestales la labor del Congreso se resume en aprobar la Ley de Presupuesto, en la que se incluyen partidas generales dentro de las cuales no se detalla la distribución táctica. Esto implica que todas las negociaciones entre el ejecutivo y el legislativo se dan de manera informal. Por esto, una vez existen las partidas generales de la Ley, hay un espacio o zona gris para la negociación entre el ejecutivo y el legislativo, y la asignación de cupos indicativos o partidas presupuestales a congresistas. Lo que complica aún más la disponibilidad de información es que esta destinación táctica del presupuesto por parte del presidente para negociar el apoyo del Congreso es inconstitucional y está abiertamente penalizada por la ley.

Dada la opacidad del proceso, la única información sobre la distribución de cupos indicativos disponible públicamente consiste en una base de datos filtrada a la prensa y publicada en el portal web Las 2 Orillas en 2014 ("La mermelada parlamentaria de puestos y contratos", 2014). Estos datos fueron dados a conocer inicialmente ante la Comisión de Acusaciones de la Cámara de Representantes por parte del representante Ernesto Macías; este último argumentó que allí se evidenciaba la asignación por parte de la Presidencia de cupos indicativos y puestos de trabajo de los cuales podían disponer los congresistas de la coalición de gobierno, en apariencia a cambio de la aprobación de proyectos importantes para el gobierno y del apoyo en la reelección del presidente en el 2014. Sin embargo, Macías nunca reveló la fuente y la Comisión de Acusaciones se declaró impedida para investigar la denuncia. La importancia única de esta información también se evidencia en que en 2018 la Corte Suprema de Justicia anunció una indagación preliminar a los congresistas teniendo como base la información de esa base de datos ("Corte Suprema de Justicia abre indagación preliminar a todo el Congreso", 2018). La Contraloría General, la entidad fiscalizadora superior del país, ha señalado que desde 2004 se han gastado en Colombia 57 billones de pesos en cupos indicativos, aunque no reveló el panel histórico de datos ("En 14 años se entregaron $\$ 57$ billones a parlamentarios por cupos indicativos: Contraloría", 2018). Lo cierto es que la ausencia de información 
y las profundas diferencias en la asignación de cupos por departamento muestran la importancia de examinar estos datos más a fondo.

Pese a estas dificultades, algunos investigadores han analizado estos datos (Valencia; Ávila, 2014; Bonilla-Mejía; Higuera-Mendieta, 2017; Rodríguez-Valero; Maldonado-Gómez, 2O2O), pero con perspectivas y objetivos distintos. Con esto en

FIGURA 1 Distribución de cupos indicativos en Colombia a nivel departamental, 2010-2014

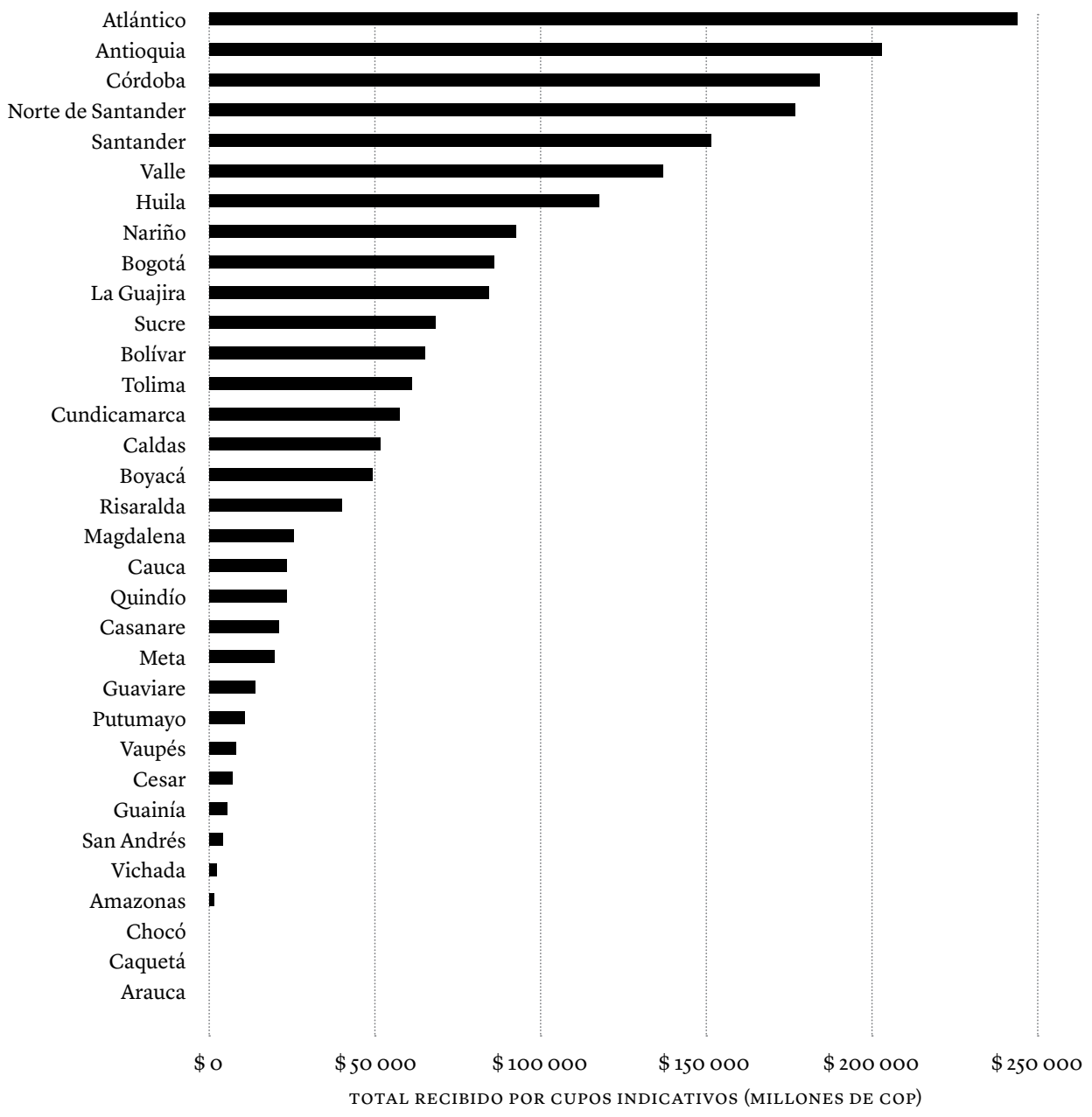


mente, extrajimos la información sobre los montos recibidos por los congresistas de la coalición del gobierno Santos por concepto de cupos indicativos de estos datos. La información filtrada solo incluía legisladores de partidos pertenecientes a la coalición de gobierno. Agregamos estos datos a nivel departamental con el fin de entender la relación entre las regiones y el centro en la distribución presupuestal. Nuestra variable dependiente es el monto total (en millones de pesos colombianos) recibido por los congresistas de cada departamento del país. La Figura 1 muestra una variación importante en los recursos recibidos por congresistas de cada departamento del país para ejecutar proyectos en esos territorios.

\section{Variables independientes}

Además de información sobre la distribución cupos indicativos a nivel departamental entre los congresistas de la coalición de gobierno Santos (2010-2014), recogimos una serie de datos a nivel departamental que nos ayudan a evaluar empíricamente las hipótesis planteadas anteriormente.

En primer lugar, recolectamos un grupo de variables de carácter demográfico y económico, las cuales permiten evaluar el gasto desde una perspectiva programática o técnica $\left(H_{1}\right)$. En este sentido, utilizamos la pobreza como una manera de medir las necesidades objetivas de los departamentos, tal y como lo proponen varios trabajos (Simison, 2014; Fried, 2012; Mejía-Guinand et al., 2008; Porto; Sanguinetti, 1996; González, 2012; González; Mamone, 2012; Nazareno et al., 2006). En particular, utilizamos el índice de necesidades básicas insatisfechas (NBI) a nivel departamental para julio de 2011, según los cálculos del Departamento Administrativo Nacional de Estadística (DANE). Este índice brinda una visión integral de las necesidades y la pobreza al integrar indicadores de ingreso, acceso a servicios y calidad de vivienda en una sola medida.

Adicionalmente, incluimos una medida del producto interno bruto (PIB) per cápita por departamento en miles de millones de pesos (base 2005). Esta variable está presente en varios análisis empíricos (Ames, 1995; Milesi-Ferretti; Peroti; Rostagno, 200o; González; Mamone, 2012; Cadot; Roller; Stephan, 2005). Aquí, utilizamos al promedio de los años 2010, 2011 y 2012 según datos del DANE, teniendo en cuenta que esta era la información disponible para los tomadores de decisiones en ese período. Esto nos permite medir el ingreso de los departamentos estudiados y dimensionar su importancia dentro de la economía del país. Además, incluimos una variable dummy para diferenciar aquellos departamentos que tenían como actividad económica principal la minería y la extracción del petróleo de aquellos que no, ya que esto podría distorsionar su PIB per cápita y estar relacionado con la distribución de gasto y presupuesto territorial. 
En segundo lugar, incluimos otro grupo de variables que permiten evidenciar si la distribución de los cupos indicativos evidencia una estrategia táctica de core voters $\left(\mathrm{H}_{2}\right)$. Para operacionalizar este concepto, identificamos las regiones donde la coalición del presidente Santos era mucho más fuerte. Utilizar resultados de las elecciones presidenciales puede conllevar problemas, ya que Santos fue elegido en 2010 con un amplio apoyo de los votantes simpatizantes del expresidente Uribe y luego, en 2014, por una coalición opuesta al uribismo. Así mismo, descartamos resultados de elecciones a gobernador, porque para este caso importa más si las mayorías regionales formaban parte o no de la coalición de gobierno, y porque los gobernadores tienen poco poder en el sistema colombiano en comparación con los congresistas de su departamento. En cambio, a partir de datos de la Registraduría Nacional, calculamos el porcentaje de alcaldes municipales pertenecientes a partidos de oposición en cada uno de los departamentos. Para el caso colombiano, Bonilla-Mejía e Higuera-Mendieta (2017) encuentran que los alcaldes alineados con el gobierno nacional reciben más transferencias discrecionales, lo cual subraya la importancia de esta variable. Además, puesto que la literatura sugiere que la estrategia de distribución hacia core voters se hace menor conforme aumenta la burocratización, incluimos el índice de desempeño integral (IDI) a nivel departamental siguiendo la medición del Departamento Nacional de Planeación (DNP). Este índice evalúa la eficacia, eficiencia y gestión del gobierno subnacional en el país con base en ejecución de presupuestos, desempeño fiscal, cumplimiento de normativas y prestación de servicios públicos.

Incluimos un tercer grupo de variables para explorar la hipótesis de representación política $\left(H_{3}\right)$; de nuevo, partimos de la estrategia empírica utilizada por trabajos existentes (Gibson; Calvo, 2001; Calvo; Murillo, 2004; González, 2012; Simison, 2014). Primero, incluimos la población de cada departamento (como porcentaje del total del país). En segundo lugar, calculamos el nivel de representación en la Cámara y el Senado colombianos por departamento. La medida corresponde al porcentaje de congresistas pertenecientes a cada departamento. En el caso de los representantes a la Cámara, su conexión con un departamento es clara: son elegidos en circunscripciones multinominales departamentales. Este sistema guarda proporcionalidad con la población departamental, con excepción de algunos departamentos (por ejemplo, Amazonas, Arauca y Vaupés) a los cuales se les asigna una representación mínima de dos congresistas a pesar de tener poblaciones muy pequeñas.

Mientras, el Senado colombiano tiene una circunscripción multinominal única de carácter nacional, lo cual dificulta asociar senadores con departamentos específicos. Sin embargo, la distribución de votos de numerosos senadores está altamente concentrada en algunos departamentos y municipios, como muestran Crisp e Ingall (2002), Basset (2015) y Milanese y Manfredi (2018). De esta manera, asociamos 
senadores a departamentos según la distribución y concentración de sus votos en las elecciones de 2010; por ejemplo, el Departamento de Antioquia cuenta con aproximadamente el $13 \%$ de la población del país, pero solo el $7 \%$ del total de los senadores en el Congreso. Además, examinamos los niveles de participación política en cada departamento, medidos como el porcentaje de votantes en las elecciones al Congreso de 2014. Niveles más altos de participación aumentan el número de curules en el Senado que puede obtener un departamento según la medida que discutimos anteriormente.

Para cerrar, y con el fin de incorporar el nivel de periferialización del gasto y entender la importancia de las estrategias tácticas y la posible configuración de una descentralización cooptativa (Gordin, 2009), medimos el nivel de dependencia de los departamentos de las transferencias a través de una de las dimensiones del índice de desempeño fiscal del DNP (peso de las transferencias con relación a los ingresos totales de los departamentos). El Cuadro 1 muestra estadísticas descriptivas para todas las variables descritas anteriormente.

CUADRO 1 Estadísticas descriptivas de las variables dependientes e independientes, 2010-2014

\begin{tabular}{|c|c|c|}
\hline Variable & Media & [Mín. - máx.] \\
\hline Total de cupos (millones de COP) & 61592,27 & [o - 243831] \\
\hline Total de cupos (millones de COP), Senado & 35250,55 & {$[0-185821]$} \\
\hline Total de cupos (millones de COP), Cámara & 26341,73 & {$[0-104139]$} \\
\hline Índice NBI & 38,19 & {$[9,2-79,19]$} \\
\hline PIB per cápita (miles de millones de COP) & 7966,40 & {$[3018,12-22608,81]$} \\
\hline Explotación minera y petrolera (indicador binario) & 0,12 & {$[0-1]$} \\
\hline Alcaldes miembros de la oposición (porcentaje) & 31,98 & {$[0-100]$} \\
\hline Índice de Desempeño Integral & 58,82 & {$[31,66-76,14]$} \\
\hline Congresistas (porcentaje) & $3, \mathrm{O} 3$ & {$[0,76-12,21]$} \\
\hline Senadores (porcentaje) & 3,03 & {$[0-13,86]$} \\
\hline Representantes (porcentaje) & 3,03 & {$[1,24-11,18]$} \\
\hline Participación electoral en legislativas (porcentaje) & 0,47 & {$[0,35-0,64]$} \\
\hline Dependencia de transferencias (porcentaje) & 67,86 & {$[26,22-89,52]$} \\
\hline Población (porcentaje) & $3, \mathrm{O} 3$ & {$[0,08-16,22]$} \\
\hline
\end{tabular}


En síntesis, esta colección de datos nos permite evaluar de forma empírica las teorías discutidas en la revisión de literatura comparada en el caso colombiano y, al mismo tiempo, sacar algunas conclusiones sobre la política distributiva en el país.

\section{Análisis: la mermelada durante el primer gobierno Santos}

¿Qué factores se asocian más con la distribución territorial de recursos y presupuesto por parte del presidente? ¿Encontramos evidencia de un tipo de distribución programática o de estrategias políticas en el caso de los cupos indicativos del gobierno Santos? En esta sección respondemos a estas preguntas analizando los datos discutidos previamente. Nuestro análisis se centra en la estimación de modelos de regresión lineal para estimar, por un lado, la relación entre variables que capturan las distintas teorías descritas en la sección anterior; y por otro, la distribución presupuestal a nivel departamental en el país.

Nuestro propósito es entender mejor la variación en la distribución territorial del presupuesto a partir de las teorías prevalentes en la literatura. Para responder estas preguntas, estimamos una serie de modelos de regresión lineal en los que la variable dependiente es el monto total por cupos indicativos recibido por los congresistas de cada departamento durante el primer gobierno Santos. Como variables independientes incluimos las medidas discutidas en la sección anterior, lo cual nos permite evaluar las tres hipótesis que derivamos de la literatura. Consignamos los resultados de este análisis en el Cuadro 2. La columna 1 (Todos) muestra resultados de un modelo en el que la variable dependiente es el total de cupos destinados a senadores y representantes de la coalición de gobierno; en la columna 2 (Senado) tomamos solo las asignaciones a senadores; y en la 3 (Cámara), solo los montos adscritos a representantes a la Cámara. En las columnas 4-6 - “Todos ( $\sin$ D. C.)", "Senado (sin D. C.)" y “Cámara (sin D. C.)"- estimamos estos mismos modelos, pero omitiendo la observación de Bogotá D. C. de la muestra, con el fin de evaluar si la inclusión del Distrito Capital (una observación atípica en términos de influencia política, calidad de vida y producción económica) afecta los resultados.

En primer lugar, discutimos el ajuste de los modelos en general. Si bien contamos con pocas observaciones $(N=33 ; 32$ departamentos más Bogotá), los modelos muestran un buen ajuste a los datos basado en los valores de la estadística $\mathrm{R}^{2}$, lo cual significa que capturamos una buena parte de la variación en la distribución del presupuesto. Adicionalmente, este ajuste no está influenciado por la observación de Bogotá D. C., pues su exclusión del análisis no afecta de forma significativa los resultados: los coeficientes y errores estándar en las columnas 1-3 son similares a los de las columnas 4-6. Por último, no hay evidencia fuerte de heteroesquedasti- 
cidad de los residuos: una prueba de Breusch-Pagan resulta en $p>0,05$ para todos los modelos, excepto para el de la columna 4. Como salvaguarda, estimamos y reportamos errores estándar consistentes con heteroesquedasticidad tipo $\mathrm{HC}_{2} \mathrm{y}$ con propiedades útiles en muestras pequeñas para todos los modelos y coeficientes (MacKinnon; White, 1985).

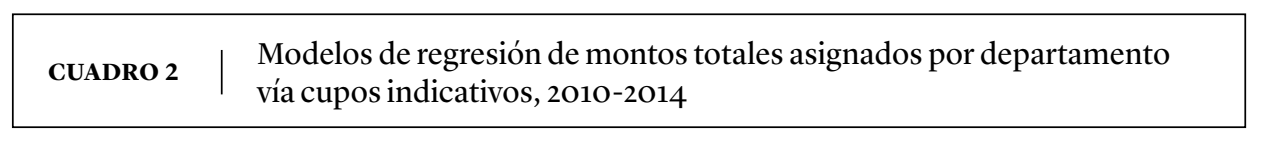

\begin{tabular}{|c|c|c|c|c|c|c|}
\hline & Todos & Senado & Cámara & $\begin{array}{c}\text { Todos } \\
\text { (sin D.C.) }\end{array}$ & $\begin{array}{c}\text { Senado } \\
(\sin \text { D. C. })\end{array}$ & $\begin{array}{l}\text { Cámara } \\
\text { (sin D. C.) }\end{array}$ \\
\hline \multirow[t]{2}{*}{ PIB per cápita } & $-1,94$ & $-2,42$ & 0,41 & $-1,68$ & $-2,31$ & 0,90 \\
\hline & $(2,78)$ & $(1,49)$ & $(1,75)$ & $(2,72)$ & $(1,47)$ & $(1,58)$ \\
\hline \multirow[t]{2}{*}{ NBI } & 1209,03 & 1004,35 & 208,65 & 1265,88 & 1044,66 & 39,17 \\
\hline & $(1043,61)$ & $(777,76)$ & $(413,88)$ & $(918,92)$ & $(725,21)$ & $(291,00)$ \\
\hline \multirow[t]{2}{*}{$\begin{array}{l}\text { Explotación } \\
\text { minera }\end{array}$} & 58496,85 & $54358,78^{* * *}$ & $-507,56$ & 50949,40 & $53015,26^{* *}$ & $-13398,80$ \\
\hline & $(34978,65)$ & $(18867,02)$ & $(21810,71)$ & $(40142,71)$ & $(21091,04)$ & $(20520,37)$ \\
\hline \multirow[t]{2}{*}{$\begin{array}{l}\text { Oposición (\% de } \\
\text { alcaldes) }\end{array}$} & $-733,18$ & $-443,78$ & $-243,35$ & $-183,18$ & $-211,01$ & 49,55 \\
\hline & $(494,94)$ & $(306,60)$ & $(217,11)$ & $(384,41)$ & $(325,63)$ & $(123,87)$ \\
\hline \multirow[t]{2}{*}{ IDI } & 252,61 & $-541,39$ & 991,80 & 530,41 & $-455,53$ & $917,77^{*}$ \\
\hline & $(1182,94)$ & $(950,54)$ & $(581,18)$ & $(1149,38)$ & $(972,54)$ & $(450,90)$ \\
\hline \multirow[t]{2}{*}{ Congresistas (\%) } & $41282,14^{* *}$ & & & $36475,59^{* *}$ & & \\
\hline & $(17544,88)$ & & & $(16335,24)$ & & \\
\hline \multirow[t]{2}{*}{ Senadores (\%) } & & $12668,58^{* *}$ & & & $12682,11^{* *}$ & \\
\hline & & $(5040,95)$ & & & $(4627,09)$ & \\
\hline \multirow[t]{2}{*}{$\begin{array}{l}\text { Representantes } \\
(\%)\end{array}$} & & & $24401,47^{*}$ & & & 9792,01 \\
\hline & & & $(13099,88)$ & & & $(9236,90)$ \\
\hline \multirow[t]{2}{*}{ Participación (\%) } & 165247,86 & 141509,62 & 71515,79 & 248931,93 & 167328,41 & $91513,35^{*}$ \\
\hline & $(167330,97)$ & $(153538,28)$ & $(46025,30)$ & $(196785,81)$ & $(171491,86)$ & $(47784,61)$ \\
\hline \multirow[t]{2}{*}{ Dependencia } & $-1926,86$ & $-2122,62^{* *}$ & 248,56 & $-1568,76$ & $-1981,66^{*}$ & 490,63 \\
\hline & $(1250,05)$ & $(987,36)$ & $(460,57)$ & $(1230,75)$ & $(995,99)$ & $(362,03)$ \\
\hline Población (\%) & $-19549,06$ & $-4490,70$ & $-11674,37$ & $-10741,79$ & $-2174,25$ & 1241,36 \\
\hline
\end{tabular}




\begin{tabular}{|c|c|c|c|c|c|c|}
\hline & $(15445,31)$ & $(6017,24)$ & $(9616,38)$ & $(12465,14)$ & $(5304,77)$ & $(6130,69)$ \\
\hline \multirow[t]{2}{*}{ (Intercepto) } & 19790,63 & 108512,64 & $-124364,90$ & $-88595,07$ & $67 \mathrm{o} 31,80$ & $-142833,11^{* *}$ \\
\hline & $(174206,36)$ & $(134554,94)$ & $(83231,18)$ & $(175$ 104,04) & $(147058,66)$ & $(66826,12)$ \\
\hline $\mathbf{N}$ & 33 & 33 & 33 & 32 & 32 & 32 \\
\hline $\mathbf{R 2}$ & 0,670 & 0,655 & 0,601 & 0,727 & 0,673 & 0,739 \\
\hline R2 ajustado & 0,540 & 0,520 & 0,445 & 0,615 & 0,539 & 0,632 \\
\hline $\begin{array}{l}\text { Nota: errores e } \\
{ }^{*} \mathrm{p}<0.1,{ }^{* *} \mathrm{p}<\end{array}$ & $\begin{array}{l}\text { r consistentes } \\
{ }^{* *} \mathrm{p}<\mathrm{O} . \mathrm{O} 1 .\end{array}$ & n heteroceda & cidad (HC2) el & aréntesis. & & \\
\hline
\end{tabular}

En cuanto a resultados sustantivos, no encontramos sustento empírico para $H_{1}$ (distribución programática) y $H_{2}$. Ninguna de las variables que operacionalizan las teorías sobre criterios objetivos $\left(H_{1}\right)$ y la distribución no programática orientada a votantes $\left(\mathrm{H}_{2}\right)$ tiene una relación estadísticamente significativa con la variable dependiente de manera consistente en todos los modelos. La evidencia no apoya la idea de que el presidente destina los recursos de los cupos para aquellos departamentos con más necesidad o en los que hay más core o swing voters, aunque sí parece que en comparación con departamentos sin explotación minera, los departamentos mineros reciben significativamente más recursos. Así, estas tesis centrales del debate sobre política distributiva en Latinoamérica y en términos comparativos no aparecen claramente en el caso colombiano. En cambio, sí encontramos evidencia que nos invita a pensar en los factores de representación y negociación $\left(H_{3}\right)$. La variable de representación en el Congreso consistentemente presenta una asociación positiva y estadísticamente significativa con los montos otorgados por departamento, tanto en total, como a senadores y representantes. En promedio, departamentos con mayores niveles de representación, medida como el porcentaje de congresistas de ese departamento, recibieron mayor cantidad de recursos por concepto de cupos indicativos. Más precisamente, ceteris paribus, un aumento de $1 \%$ en la representación total de un departamento se asocia con un aumento de aproximadamente 41,282 millones de pesos colombianos en los cupos indicativos dirigidos hacia los congresistas de ese departamento. Teniendo en cuenta el rango de ambas variables (ver Cuadro 1), la magnitud de este coeficiente no es solo estadísticamente significativa, sino sustantivamente importante. En general, este hallazgo está en sintonía con trabajos anteriores como el de Mejía-Guinand et al. (2008), quienes sostienen que las consideraciones políticas primaban sobre las técnicas en la asignación de recursos para la construcción de vías en el país. 
La Figura 2 permite visualizar estos resultados con más claridad. Aquí, mostramos el efecto marginal de un aumento en la representación legislativa (en Senado y Cámara) sobre los montos recibidos a nivel departamental por concepto de cupos indicativos. Ceteris paribus, pasar de $2 \%$ a $5 \%$ de representación en el Congreso se asocia con un aumento de aproximadamente 123,846 millones de pesos en cupos indicativos. Esta es una diferencia estadísticamente significativa y sustancial: solo 6 departamentos recibieron sumas más altas en este periodo. El análisis nos lleva a concluir que los departamentos con más Representantes en el Congreso recibieron más recursos por parte del presidente, y que un aumento en la representación se asocia fuertemente con aumentos importantes en la recepción de recursos. En términos teóricos, encontramos evidencia a favor de $\mathrm{H}_{3}$ : más representación política en el legislativo implica más distribución y recepción de presupuesto. Nuestro análisis apunta a una distribución táctica del presupuesto en sistemas presidencialistas y unitarios como el colombiano.

Finalmente, tras este análisis empírico agregado, es preciso explicar cómo esta distribución puede ser estratégica para un presidente que quiere consolidar una mayoría parlamentaria. En concreto, para entender la importancia del uso táctico

FIGURA 2 Efectos marginales de la representación en Cámara sobre la distribución de presupuesto por departamento, 2010-2014

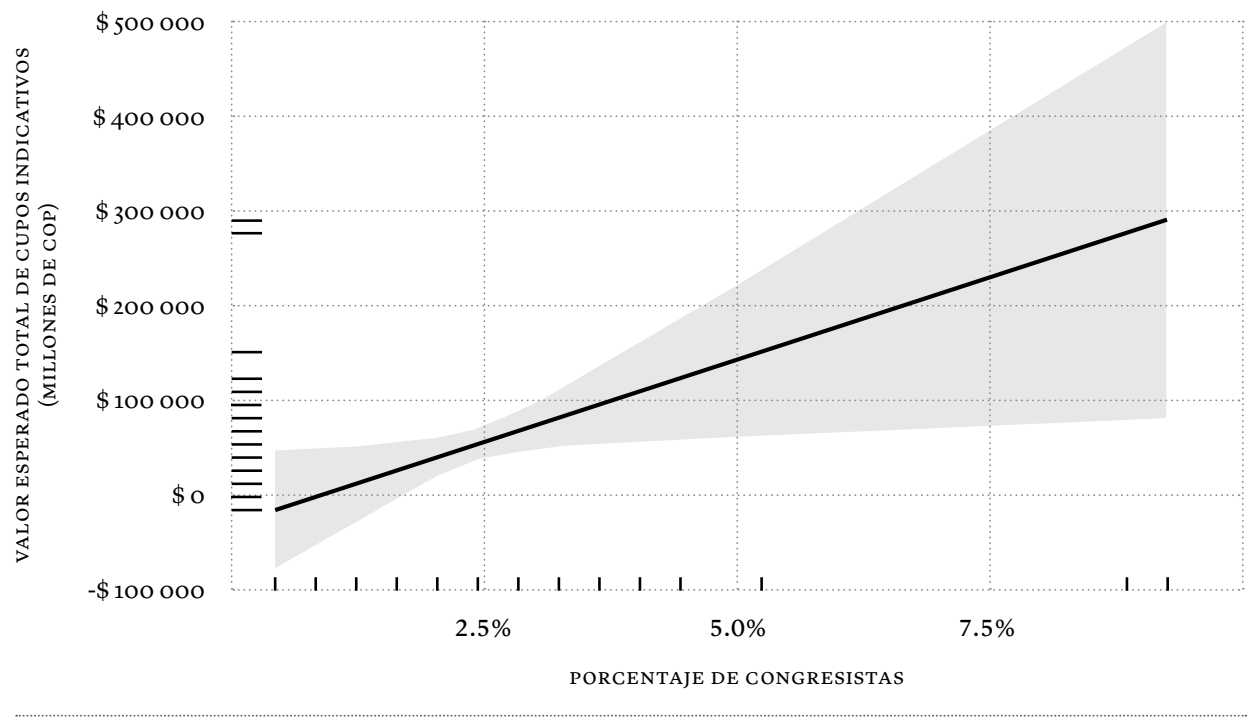


de recursos con preferencia hacia departamentos más representados en el Congreso es importante explorar la importancia que estos recursos tuvieron durante la presidencia de Santos para formar una coalición política en el Congreso. Como lo señala Simison (2014), el deslinde entre ejecutivo y legislativo en los regímenes presidencialistas dificulta la creación de coaliciones estables, por lo cual la distribución no programática de recursos se vuelve una estrategia importante. En el contexto colombiano, Pachón-Buitrago y Carroll (2018) hacen énfasis en la dificultad de construir coaliciones ejecutivo-legislativo y de traducirlas en ventajas legislativas.

Frente a estas dificultades, el presidente Santos tenía la tarea de construir una coalición partiendo de su ruptura con Uribe en 2010, lo cual lo dejaba con menos aliados en el Congreso. Además, lo debía hacer en el marco de un período breve antes de la campaña electoral y la reelección de 2014. Esta situación fue ilustrada por el jurista colombiano Rodrigo Uprimny en una entrevista radial:

(...) una vez elegido un congresista, él tiene su período fijo de 4 años y su suerte no depende mucho de la del gobierno, (...) puede distanciarse políticamente del gobierno, y el gobierno no puede hacer mucho porque no puede disolver el parlamento. Esa separación orgánica estricta entre el congreso electo por un período, cuando no es un régimen bipartidista, sino multipartidista, [lleva a que los gobiernos no tengan] mayorías en el congreso, [y tengan que recurrir] a distintas estrategias, a veces ideológicas, para tratar de configurar una mayoría ideológica, pero eso muchas veces no funciona, entonces recurren a este tipo de prácticas [los cupos indicativos] para desbloquear y hacer avanzar proyectos. (Hora 20, 2018)

De igual modo, más allá del estilo propio de Santos, esta situación de fragmentación de la coalición ideológica, dependencia de maquinarias y caída de la popularidad también genera incentivos para cambiar la canasta y distribuir más bienes privados, en vez de públicos. Precisamente, los cupos indicativos cumplen con las características de un bien privado, pues son excluyentes y desincentivan a los polizones o free-riders (Olson, 1965). De nuevo, esto coincide con lo planteado en el modelo de distribución no programática orientada hacia aquellos territorios con mayor representación política en el legislativo $\left(H_{3}\right)$.

\section{Conclusión}

En este artículo exploramos las teorías principales para explicar la distribución de los presupuestos según criterios políticos, a través del análisis de las partidas presupuestales asignadas de manera discrecional por el presidente en el Congreso colombiano, llamadas cupos indicativos. Empezamos revisando la literatura con el 
fin de trazar las principales líneas de pensamiento y derivar una serie de hipótesis empíricamente evaluables. Posteriormente, realizamos un análisis estadístico de la distribución departamental de montos totales por concepto de cupos indicativos entre miembros de la coalición del primer gobierno de Juan Manuel Santos (20102014). Encontramos que la representación de un departamento en el Congreso se asocia positiva y significativamente con una mayor recepción de esos recursos por parte de miembros de la coalición de gobierno. Esta evidencia apoya las teorías enfocadas en la distribución táctica legislativa del presupuesto. A la vez, no encontramos soporte empírico para las teorías que sugieren una distribución programática $\mathrm{u}$ orientada hacia los votantes.

Estos hallazgos empíricos permiten intuir que la distribución de cupos indicativos se convirtió en un mecanismo privilegiado de negociación entre el presidente y el legislativo, el cual se orientaba hacia los departamentos con un alto número de congresistas en relación con su población. Conjeturamos que, con esta estrategia, el gobierno buscaba lograr la aprobación de proyectos estratégicos y, quizás, la reelección presidencial en 2014. Así mismo, una implicación de nuestro trabajo es que las reformas orientadas a aumentar la representación política en el legislativo de ciertas regiones pueden tener efectos sobre la distribución de recursos y, por tanto, son una herramienta poderosa con efectos más allá de la representación simbólica.

En un contexto como el colombiano y otros similares en Latinoamérica, este hallazgo aporta en la comprensión de un fenómeno de gran importancia. Más que seguir criterios técnicos u objetivos, o de estar sujeto a las dinámicas electorales de la lucha por votantes (sean core o swing), la distribución no programática o táctica de recursos del presidente responde a una lógica política en el legislativo. Nuestros hallazgos empíricos nos permiten comenzar a entender los cálculos políticos que realizan los presidentes al distribuir recursos; ilustramos esto con el caso de la distribución de cupos indicativos en Colombia.

En este escenario, se evidencia la continuación de una historia de mecanismos de distribución discrecional de recursos que dan cuenta de una necesidad del centro de establecer nexos con los políticos regionales y de valerse de su apoyo para mantener ciertos niveles de orden en los territorios; esto ha llevado a que se entreguen auxilios parlamentarios y se concentren fondos de cofinanciación, entre otras formas de llevar presupuestos a regiones (en especial, a aquellas pobres) con baja presencia del Estado, donde con cantidades relativamente pequeñas de dinero se pueden lograr importantes réditos políticos. La evidencia que mostramos y la literatura que revisamos sugieren que este accionar se debe a un comportamiento estratégico del presidente: al percibir un mayor peso en los departamentos más representados en el Congreso, decide otorgarles más recursos por cupos indicativos a sus legisladores. 
En el marco de la distribución táctica, este artículo también permite abrir algunas preguntas adicionales sobre las estrategias políticas del presidente Santos, dada su necesidad de conformar una nueva coalición después de romper con la coalición uribista. Sobre lo anterior, se debe recordar que la decisión de llevar a cabo el proceso de paz con las FARC le implicó a Santos la ruptura con el presidente Uribe. En tal sentido, esto llevó a Santos a perder el manejo de la coalición de derecha que lo apoyó para lograr su primera presidencia y que llevaba ocho años en el gobierno. Lo anterior, en un entorno donde era posible la reelección presidencial, obligó al presidente a buscar la conformación de una nueva coalición, sobre la base de la búsqueda de la paz, pero también sobre incentivos materiales que le permitieran unir a su apuesta diferentes clientelas.

Finalmente, es importante advertir que entender la manera en que se ha utilizado el presupuesto en Colombia de manera táctica para mejorar la gobernabilidad y asegurar éxitos electorales, cobra especial relevancia en el contexto actual. El presidente Iván Duque (2018-2022) se ha propuesto acabar con este tipo de distribuciones presupuestales de tipo político, que él asocia en su discurso con la corrupción. Esta estrategia de apolitización aparente de la distribución del presupuesto le ha representado problemas para aprobar algunos de sus proyectos claves en el congreso, tales como el Plan de Desarrollo, la reforma tributaria y sus objeciones a la Justicia Especial para la Paz (JEP). Lo anterior pone de manifiesto la necesidad de entender con más profundidad el papel que juegan las estrategias de intermediación y negociación con el Congreso mediante la distribución del presupuesto.

\section{Referencias}

Ames, Barry (1995). Strategy under Open-List Proportional Representation. American Journal of Political Science, 39(2), 406-433. https://doi.org/10.2307/2111619

Aprobada la reforma a las regalías (9 de junio de 2011). El Espectador. Recuperado de https:// www.elespectador.com/content/aprobada-la-reforma-las-regal\% $3 \%$ ADas

Basset, Yann (2015). ¿Whipped Vote?: Personalization of Voting Behavior and Electoral Paths of Colombian Senators. Revista de Ciencia Política, 35(3), 489-507. http://dx.doi.org/10.4067/ So718-090X2015000300002

Benson, Allison L. (2021). From targeted private benefits to public goods: Land, distributive politics and changing political conditions in Colombia. World Development, 146. https:// doi.org/10.1016/j.worlddev.2021.105571 
Blaydes, Lisa (2020). Distributive Politics in the Middle East. En Routledge Handbook of Middle East Politics (pp. 471-479), editado por Larbi Sadiki. Abingdon: Routledge.

Bonilla-Mejía, Leonardo; Higuera-Mendieta, Iván Gonzalo (2017). Political alignment in the time of weak parties: electoral advantages and subnational transfers in Colombia. Documentos de Trabajo sobre Economía Regionaly Urbana, 260.

Cadot, Oliver; Roller, Lars-Hendrick; Stephan, Andreas (2005). Contribution to productivity or pork barrel? The two faces of infrastructure investment. Journal of Public Economics, 9o(6-7), 1133-1153. https://doi.org/10.1016/j.jpubeco.2005.08.006

Calvo, Ernesto; Murillo, María Victoria (2004). Who Delivers? Partisan Clients in the Argentine Electoral Market. American Journal of Political Science, 48(4), 742-757. https:// doi.org/10.1111/j.0092-5853.2004.00099.x

Catalinac, Amy; Bueno de Mesquita, Bruce; Smith, Alastair (2020). A Tournament Theory of Pork Barrel Politics: The Case of Japan. Comparative Political Studies, 53(10-11), 1619-1655. https://doi.org/10.1177/o010414019897677

Corte Suprema de Justicia abre indagación preliminar a todo el Congreso (13 de febrero de 2018). El Espectador. Recuperado de https://www.elespectador.com/noticias/judicial/ corte-suprema-de-justicia-abre-indagacion-preliminar-a-todo-el-congreso/

Cox, Gary (2010). Swing voters, core voter and distributive politics. En Political Representation (pp. 342-357), editado por Ian Shapiro; Susan C. Stokes; Elisabeth J. Wood; Alexander S. Kirshner. New York: Cambridge University Press. https://doi.org/10.1017/CBO9780511813146.015

Cox, Gary; McCubbins, Matthew (1986). Electoral politics as a redistributive game. Journal of Politics, 48(2), 370-389. https://doi.org/10.2307/2131098

Crisp, Brian; Ingall, Rachel (2002). Institutional Engineering and the Nature of Representation: Mapping the Effects of Electoral Reform in Colombia. American Journal of Political Science, 46(4), 733-748. https://doi.org/10.2307/3088430

Díaz-Cayeros, Alberto; Estévez, Federico; Magaloni, Beatriz (2016). The Political Logic of Poverty Relief: Electoral Strategies and Social Policy in Mexico. New York: Cambridge University Press. https://doi.org/10.1017/CBO9781316492710

En 14 años se entregaron $\$ 57$ billones a parlamentarios por cupos indicativos: Contraloría (7 de junio de 2018). El Espectador. Recuperado de https://www.elespectador.com/noticias/ judicial/en-14-anos-se-entregaron-57-billones-a-parlamentarios-por-cupos-indicativoscontraloria/

Fried, Brian (2012). Distributive Politics and Conditional Cash Transfers: The Case of Brazil's Bolsa Familia. World Economics, 4O(5), 1042-1053. https://doi.org/10.1016/j. worlddev.2011.09.022 
Garay, Candelaria; Palmer-Rubin, Brian; Poertner, Mathias (2020). Organizational and Partisan Brokerage of Social Benefits: Social Policy Linkages in Mexico. World Development, 136, 1-12. https://doi.org/10.1016/j.worlddev.2020.105103

Gibson, Edward (2012). Boundary Control: Subnational Authoritarianism in Federal Democracies. New York: Cambridge University Press. https://doi.org/10.1017/CBO9781139017992

Gibson, Edward; Calvo, Ernesto (2001). Federalism and Low Maintenance Constituencies: Territorial Dimensions of Economic Reform in Argentina. Studies in Comparative International Development, 35(3), 32-55. https://doi.org/10.1007/BFo2699765

Gibson, Edward; Calvo, Ernesto; Falleti, Tulia (2004). Reallocative Federalism: Territorial Overrepresentation and Public Spending in the Western Hemisphere. En Federalism and Democracy in Latin America (pp. 173--196), editado por Edward Gibson. Baltimore: Johns Hopkins University Press.

Golden, Miriam; Min, Brian (2013). Distributive Politics around the World. Annual Review of Political Science, 16, 73-99. https://doi.org/10.1146/annurev-polisci-052209-121553

González, Lucas (2012). The redistributive effects of centralization and decentralization across subnational units. Latin American Research Review, 47(3), 109-133. https://doi.org/10.1353/ lar.2012.0046

González, Lucas; Mamone, Ignacio (2012). La economía política de la distribución federal de obra pública en Argentina. Desarrollo Económico, 51(201), 59-80.

González, Lucas; Mamone, Ignacio (2015). Who Distributes? Presidents, Congress, Governors, and the Politics of Distribution in Argentina and Brazil. Revista Ibero-americana de Estudos Legislativos, 4, 17-32. https://doi.org/10.1266o/riel.v4.n1.2015.49203

González-Tule, Luis; Guzmán-Mendoza, Carlos Enrique; Tuirán-Sarmiento, Ángel (2021). Clientelismo personalizado en Colombia: una aproximación histórico-institucional a un estudio de caso. Revista Sociedade e Cultura, 24. https://doi.org/10.5216/sec.v24.57225

Gordin, Jorge P. (2009). The Politics of Fiscal Decentralization Revisited: A Typology and Comparative Evidence. Fédéralisme Régionalisme, 9(2), 1-13.

Hicken, Allen (2011). Clientelism. Annual Review of Political Science, 14, 289-310. https://doi. org/10.1146/annurev.polisci.031908.220508

Hicken, Allen; Nathan, Noah L. (2020). Clientelism's Red Herrings: Dead Ends and New Directions in the Study of Nonprogrammatic Politics. Annual Review of Political Science, 23, 277-294. https://doi.org/10.1146/annurev-polisci-050718-032657

Hora 20 (14 de febrero de 2018). Caracol Radio [programa de radio]. Recuperado de https:// alacarta.caracol.com.co/audio/o97RD21000oooo641717/ 
Horiuchi, Yusaku; Lee, Seungjoo (2008). The Presidency, Regionalism, and Distributive Politics in South Korea. Comparative Political Studies, 41(6), 861-8-82. https://doi. org/10.1177/0010414006298900

Jiang, Junyan; Zhang, Muyang (2020). Friends with benefits: Patronage networks and distributive politics in China. Journal of Public Economics, 184. https://doi.org/10.1016/j. jpubeco.2020.104143

La mermelada parlamentaria de puestos y contratos (2014). Las 2 Orillas. Recuperado de http:// www.las2orillas.co/la-mermelada-parlamentaria-de-puestos-contratos/

Larcinese, Valentino; Snyder, James M.; Testa, Cecilia (2013). Testing Models of Distributive Politics using Exit Polls to Measure Voters' Preferences and Partisanship. British Journal of Political Science, 43(4), 845-875. https://doi.org/10.1017/Sooo7123412000245

Lee, Frances (2000). Senate Representation and Coalition Building in Distributive Politics. American Political Science Review, 94(1), 59-72. https://doi.org/10.2307/2586380

Leyva-Botero, Santiago (2012). Las relaciones intergubernamentales en Colombia (2002-2010): Un análisis de los Consejos Comunales de gobierno. Análisis Político, 25(76), 125-144.

MacKinnon, James G.; White, Halberd (1985). Some heteroskedasticity consistent covariance matrix estimators with improved finite sample properties. Journal of Econometrics, 29(3), 305-325. https://doi.org/10.1016/0304-4076(85)90158-7

Mebane, Walter; Wawro, Geoffrey (2002). Presidential Pork Barrel Politics. Inédito.

Mejía-Guinand, Luis Bernardo; Botero, Felipe; Rodríguez-Raga, Juan Carlos (2008). ¿Pavimentando con votos? Apropiación presupuestal para proyectos de infraestructura vial en Colombia, 2002-2006. Colombia Internacional, 69, 14-42. https://doi.org/10.7440/ colombiaint68.2008.01

Milanese, Juan Pablo; Manfredi, Luciana (2018). Nationalization of the Legislative Vote, Visibility and Reputation in the Written Press: An Analysis of Candidates' Strategies for the 2014 Colombian Senate Election. Journal of Iberian and Latin American Research, 24(3), 199-213. https://doi.org/10.1080/13260219.2019.1623289

Milesi-Ferretti, Gian Maria; Perotti, Roberto; Rostagno, Massimo (200o). Electoral Systems and Public Spending. The Quarterly Journal of Economics, $117(2), 609-657$. https://doi. org/10.1162/o03355302753650346

Nazareno, Marcelo; Stokes, Susan C.; Brusco, Valeria (2006). Réditos y peligros electorales del gasto público en la Argentina. Desarrollo Económico, 46(181), 63-88. https://doi. org/10.2307/4151101

Olson, Mancur (1965). The Logic of Collective Action: Public Goods and the Theory of Groups. Cambridge: Harvard University Press. 
Pachón-Buitrago, Mónica; Carroll, Royce (2016). The Unrealized Potential of Presidential Coalitions in Colombia. En Legislative Institutions and Lawmaking in Latin America (pp. 122-147), editado por Eduardo Alemán; George Tsebelis. Oxford: Oxford University Press. https://doi.org/10.1093/acprof:oso/9780198777861.001.0001

Porto, Alberto; Sanguinetti, Pablo (1996). Las transferencias intergubernamentales y la equidad distributiva: el caso argentino. Santiago de Chile: Comisión Económica para América Latina y el CaribeCepal.

Porto, Alberto; Sanguinetti, Pablo (2001). Political determinants of intergovernmental grants: Evidence from Argentina. Economics \& Politics, 13(3), 237-256. https://doi.org/10.1111/14680343.00092

Raile, Eric D.; Pereira, Carlos; Power, Timothy J. (2011). The Executive Toolbox: Building Legislative Support in a Multiparty Presidential Regime. Political Research Quarterly, 64(2), 323-334. https://doi.org/10.1177/1065912909355711

Rodríguez-Valero, Luis Alfredo; Maldonado-Gómez, Néstor (2020). Las políticas distributivas en Colombia: de los auxilios parlamentarios a las partidas de inversión social regional o 'mermelada tóxica'. Revista de la Facultad de Derecho y Ciencias Políticas, 5o(132), 1-28. https://doi.org/10.18566/rfdcp.v50n132.ao1

Rogoff, Kenneth (1991). Equilibrium Political Budget Cycles. The American Economic Review, $80(1), 21-36$.

Simison, Emilia (2014). Estrategias distributivas presidenciales en contextos cambiantes de centralización fiscal y nacionalización partidaria: Transferencias discrecionales en Argentina durante presidencias peronistas. Revista de Ciencia Política, 34(3), 561-582. http:// dx.doi.org/10.4067/So718-090X2014000300003

Stokes, Susan C. (2005). Perverse Accountability: A Formal Model of Machine Politics with Evidence from Argentina. American Political Science Review, 99(3), 315-325. https:/doi. org/10.1017/Sooo3055405051683

Stokes, Susan; Dunning, Thad; Nazareno, Marcelo; Brusco, Valeria (2013). Brokers, Voters and Clientelism: The Puzzle of Distributive Politics. New York: Cambridge University Press. https://doi.org/10.1017/CBO9781107324909

Valencia, León; Ávila, Ariel (2014). Herederos del mal. Clanes, mafias y mermelada. Congreso 2014-2018. Bogotá: Ediciones B. 